\title{
Network Performance Evaluation of Abis Interface over DVB-S2 in the GSM over Satellite Network
}

\author{
S. B. Musabekov, ${ }^{1,2}$ P. K. Srinivasan, ${ }^{3}$ A. S. Durai, ${ }^{3}$ and R. R. Ibraimov ${ }^{4}$ \\ ${ }^{1}$ ZTE Investment LLC, Oybek Street 14, Tashkent 100015, Uzbekistan \\ ${ }^{2}$ Ahmadabad Earth Station, Space Applications Centre (SAC), Headquarters, 4 Kalidas Road, Dehradun, 248001, India \\ ${ }^{3}$ Indian Space Research Organization (ISRO), Ahmedabad, 380015, India \\ ${ }^{4}$ Tashkent University of Information Technology (TUIT), 108, Amir Temur Street, Tashkent 100084, Uzbekistan
}

Correspondence should be addressed to S. B. Musabekov, smusabekov@yahoo.com

Received 30 September 2009; Revised 14 May 2010; Accepted 21 July 2010

Academic Editor: George Xilouris

Copyright (c) 2010 S. B. Musabekov et al. This is an open access article distributed under the Creative Commons Attribution License, which permits unrestricted use, distribution, and reproduction in any medium, provided the original work is properly cited.

\begin{abstract}
This paper deals with establishing a GSM link over Satellite. Abis interface, which is defined between Base Transceiver Station (BTS) and Base Station Controller (BSC), in a GSM network is considered here to be routed over the Satellite. The satellite link enables a quick and cost-effective GSM link in meagerly populated areas. A different scenario comparison was done to understand the impact of Satellite environment on network availability comparing to terrestrial scenario. We have implemented an Abis interface over DVB S2 in NS2 and evaluated the performance over the high delay and loss satellite channel. Network performance was evaluated with respect to Satellite channel delay and DVB S2 encapsulation efficiency under different amount of user traffic and compared with the terrestrial scenario. The results clearly showed an increased amount of SDCCH and TCH channels required in the case of satellite scenario for the same amount of traffic in comparison to conventional terrestrial scenario. We have optimized the parameters based on the simulation results. Link budget estimation considering DVB-S2 platform was done to find satellite bandwidth and cost requirements for different network setups.
\end{abstract}

\section{Introduction}

The success story of second-generation $(2 \mathrm{G})$ terrestrial mobile systems (GSM) and the relative demise of $2 \mathrm{G}$ mobile satellite systems (MSS) such as, Iridium and Globalstar have influenced the future of MSS. These two distinct but interrelated events demonstrate the importance of proper market and business strategies for the success of the future mobile satellite industry. Global System for Mobile communications (GSM) is the most popular means for voice and data communication having more than 2 billion subscribers all over the world. Still 3/4 of the globe is not covered by GSM networks. Despite growing demand for GSM services in rural areas, it is not cost-effective for GSM service providers to cover areas with meager population density. Poor terrestrial infrastructure in remote areas leads to high capital expenditures for establishing new links by means of fiber optic cables or microwave links, leading to an alternate and cost-effective solution like Satellite interface. Proposed work considers DVB-S2 [1] as a physical interface between Earth station and Satellite due to its highly spectrum efficient Modulation and powerful FEC schemes (ModCode). DVBS2 has two different frames, long (64800 bit) and short (16200 bit) frames. Hence encapsulation efficiency and Network bandwidth utilization should be evaluated for different scenarios.

Presently there are no clear specifications on Abis interface over satellite technology. Still there are many proprietary solutions present at the world market. There is a lack of open standard definition in this area. Issues like change in signaling protocol on Abis interface while routing through Satellite are not addressed. Questions about implications on network availability and integrity while switching to GSM over satellite technology are not discussed elsewhere, which lead to a definition of an open standard architecture for an Abis interface over Satellite. We had proposed in [2] a novel 


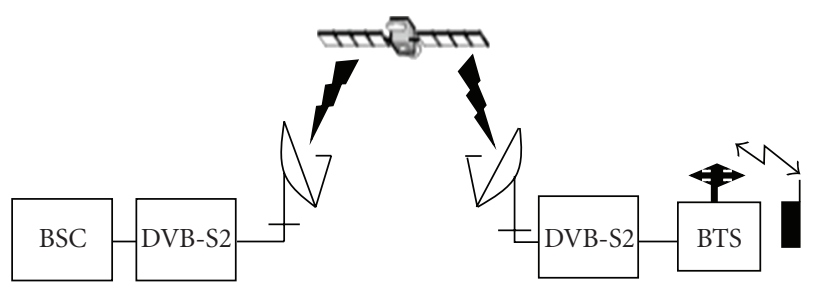

Figure 1: Abis interface over Satellite [4].

OSI architecture for Abis over satellite interface but there is a requirement of network performance evaluation, which is attempted in this paper.

We have proposed a new protocol architecture called Abis over IP over DVB-S2 in which the signaling and Transcoding Rate and Adaptation Unit (TRAU) frames are formatted over $\mathrm{UDP} / \mathrm{IP}$ and RTP/UDP/IP, respectively, and encapsulated in Generic Stream (GS) stream of DVB-S2 over the forward and return link. Simulation is performed using standard network simulator NS-2.33 [3] under delay and different loss scenarios of the Satellite and the results are analyzed.

The following sections are organized as follows. Section 2 provides NS2 network (OSI) model; Section 3 gives the mathematical analysis for the traffic, subscriber density, and BTS capacity requirements for Abis over Satellite and terrestrial Abis. Section 4 describes Simulation Parameters, Results, and Analysis. Section 5 presents link budget estimations for Abis over DVB-S2 platform. Section 6 concludes this paper.

\section{Proposed Abis over IP over DVB-S2 Network Performance Evaluation}

The system setup for Abis interface over IP over DVB-S2 is shown in Figure 1, where the proposed protocol architecture lies between the BTS and BSC.

Figure 2 shows the proposed signaling protocol architecture for Abis interface over Satellite, and Figure 3 shows framing format. Frames on Abis interface are separated into TRAU frames and Signaling frames. The signaling frames is formatted over UDP/IP and encapsulated into Data Field Length (DFL) of DVB-S2 frame. The traffic in TRAU frames are formatted over RTP/UDP/IP and encapsulated over DVB-S2. Before IP encapsulation timeslot elimination technique may be applied to save bandwidth to considerable amount [5].

In the forward link, that is, from BSC to BTS, these messages describe the link establishment and release information and its acknowledgment to all its BTS [6]. The messages with added UDP and IP header form a multiple transport stream of the DVB-S2 system. The Base Band (BB), FEC and Physical Layer (PL) headers of DVB-S2 frame are added and modulated before given to the RF Satellite channel. The signaling links over the Abis interface are addressed to the different units by Terminal Endpoint Identifiers (TEIs) [7]. UDP header will represent destination port address of LAPD frame. Each of the physical link time slots of E1 [8] is now

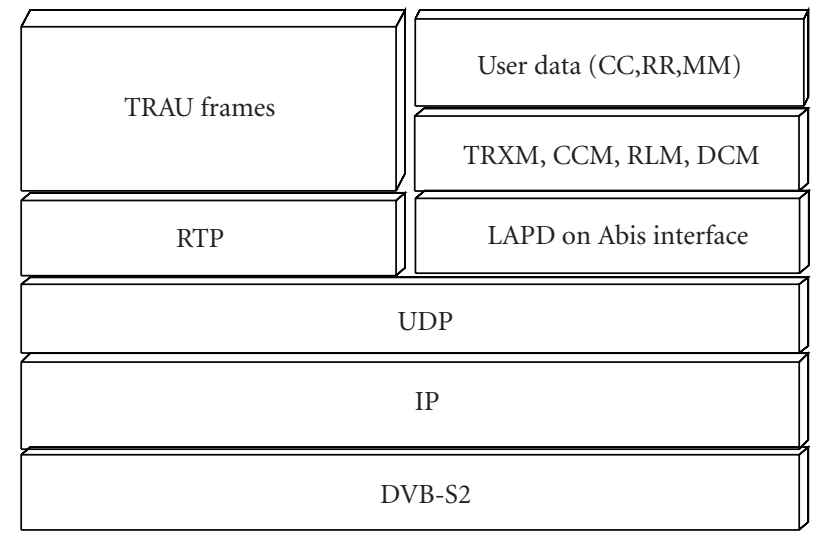

FIGURE 2: Abis interface over Satellite protocol architecture.

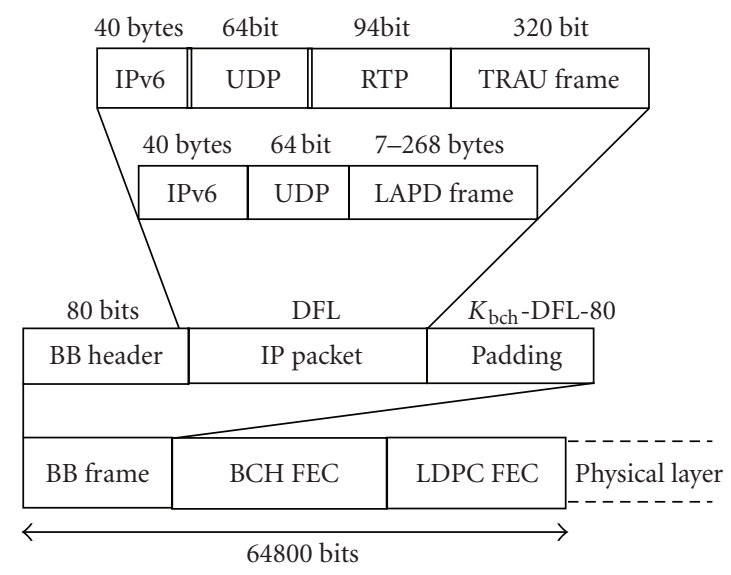

FIgURE 3: Framing format.

distinguished by each stream of the multiplexed GS stream fed to the DVB-S2 system.

TRAU frames [9] which carry voice are encapsulated into Real-Time Protocol (RTP) packets with time stamp of playback to prevent jitter while receiving, then encapsulated into UDP packets. Resulting UDP packets are IP encapsulated and formed Generic stream for the DVB-S2 system. DVB-S2 has inherent bandwidth efficient modulation modes and power efficient coding.

\section{Traffic Analyses}

This section gives the mathematical analysis for the traffic and subscriber density for the satellite scenario compared with the terrestrial scenario [10]. Each BTS has three sectors (cells). Each cell has one TRX containing one time slot (TS) for BCCH (Broadcasting Control Channel) to broadcast information about serving BTS, SDCCH (Stand Alone Dedicated Control Channel) for signaling during MOC, MTC, and Location Update, FACCH (Fast Associated Control Channel) for transferring measurements results and handover. Other TRX within this cell will have only TCH. If the number of TRX is more than three, then one more SDCCH TS should be added. Traffic refers to the numbers 
of subscribers the network can support and is described as follows:

$$
A=n \times \frac{T}{3600},
$$

where $n$-Calls are made by a subscriber within an hour, $T$ is Average duration of each call (in seconds), and $A$ is Traffic, in Erlang.

If one call is made by a subscriber within an hour and last 120 seconds, the traffic is calculated as $A=1 \times 120 / 3600=$ $33 \mathrm{mErl}$. For convenience of engineering calculation, the traffic is defined as $25 \mathrm{mErl}$ per subscriber. The SDCCH average process time for MOC, MTC is considered as 3 seconds. Location updating process takes 9 seconds, BHCA (Busy Hour Call Attempts) $=2$.

The traffic of SDCCH per subscriber is

$$
\frac{3 \times 2+9}{3600}=0.0042 \text { Erlang. }
$$

For 4 SDCCH and blocking probability of $2 \%$, we can support 1.092 Erlang (from Erlang B table). SDCCH/8 has $8 \mathrm{SDCCH}$ logical channels within one time slot. Hence,

$$
\left(\frac{1.092}{0.0042}=260 \mathrm{sub}\right) \times 0.025 \text { Erlang }=6.5 \text { Erlang. }
$$

In Erlang-B with blocking probability of 2\%, 6.5 Erlang needs 12TCH (2TRX). During the establishment and terminating of MOC and MTC, 29 commands and response I frames are transferred between BTS and BSC. Each frame will be delayed by $t \approx 240 \mathrm{~ms}$ while propagating through Satellite. For Satellite communication, SDCCH average process time for MOC and MTC approximately will be 7 seconds due to Satellite delay; location updating process will be 20 seconds. Assuming $2 \mathrm{BHCA}$, we have the traffic of SDCCH per subscriber as

$$
\frac{7 \times 2+20}{3600}=0.0094 \text { Erlang. }
$$

By 4SDCCH with blocking probability of $2 \%$, we can support 1.092 Erlang (from Erlang B table).

$$
\left(\frac{1.092}{0.0094}=116 \mathrm{sub}\right) \times 0.025 \mathrm{Erl}=2.9 \mathrm{Erl} .
$$

In Erlang-B with blocking probability of 2\%, 2.9 Erlang need 7 TCH (1TRX) channels.

From above calculations, it can be concluded that for Abis over Satellite the same amount of Erlangs on SDCCH channel can support less number of subscribers than in terrestrial communication.

The same amount of subscribers is taken into account for Satellite Abis and terrestrial Abis 200 subscribers. Every time when MS initiates a call, there will be delay during call setup and also during conversation. Since each message signaling and traffic will be delayed while sending over satellite channel, it is considered that after call set-up phase subscriber needs to deliver 40 messages, and message

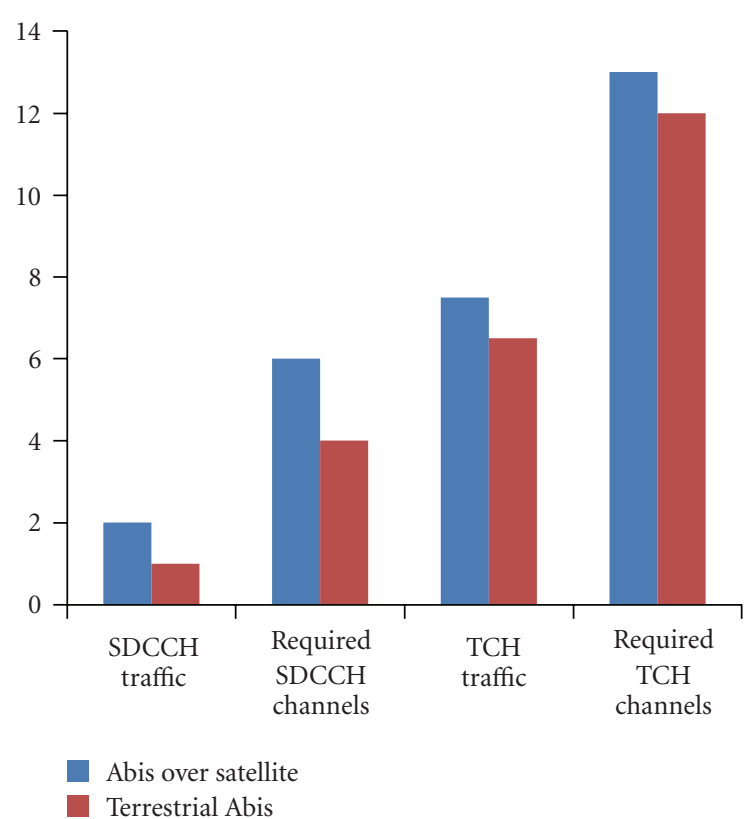

FIgURE 4: Traffic for Abis over Satellite and Terrestrial Abis.

duration is 3 seconds. Hence one conversation time will be $40 * 3=120$ seconds. In case of Abis over Satellite, conversation time will increase to 129.6 seconds. Hence mErl per subscriber will increase. Figure 4 shows comparison of Satellite Abis and Terrestrial Abis for the above described scenario. This shows that to support same amount of traffic a number of SDCCH and TCH channels are required in Abis over Satellite scenario. This calculation will give the number of TRX's or TS required for the same amount of the traffic.

\section{Simulations}

4.1. Simulation Description and Parameters. Simulation of the proposed OSI stack was performed using the NS version 2.33. The LAPD generation was based on the source code found under over which the rest of the OSI stack is incorporated. During simulation, two signaling frames from Um interface are considered Channel request and Connection acknowledgment messages. SABM frame to BSC to establish signaling link between BSC and BTS and sent over UDP/IP and over DVB-S2 frame. Corresponding UA frame will be sent to BTS. When LAPD link is established, Chan_Req message will be forwarded and 27 consecutive command response frames are sent in both directions to simulate call setup scenario. Call is established when connection acknowledgment frame is received. Table 1 shows simulation parameters.

Simulation is done for various scenarios where configuration of BTS varied from 1 TRX to 12 TRX. Each scenario considers different number of users and data rates for Abis interface and Satellite channel. Only CS traffic is considered during simulation over the fixed DVB-S2 frames. Simulation is done to monitor the encapsulation efficiency of Abis interface over IP over DVB-S2 platform. 
TABLE 1: Simulation parameters.

\begin{tabular}{lcc}
\hline Sr. No & Simulation parameter & Value \\
\hline 1 & No. of MS/BTS & $1-150$ \\
2 & No. of time slots/BTS & $8-168$ \\
3 & Call duration & $120 \mathrm{sec}$ \\
4 & N200 & 3 \\
5 & T200 & $200 \mathrm{~ms}-900 \mathrm{~ms}$ \\
6 & I-Frame length & $7-268$ bytes \\
7 & Data rate for one voice channel & $16 \mathrm{Kbps}$ \\
8 & DVB-S2 frame & $64800 / 16200 \mathrm{bits}$ \\
9 & DVB-S2 FEC & $3 / 4$ \\
10 & DVB-S2 DFL & $48408 / 11712 \mathrm{bits}$ \\
11 & Channel delay & $250 \mathrm{msec}$ \\
12 & Channel loss Probability & $93 \% \mathrm{Good}, 7 \% \mathrm{Bad}$ \\
\hline
\end{tabular}

4.2. Simulation Results. Three scenarios are considered with different timer T200 [11] values of $200 \mathrm{~ms}$ and $900 \mathrm{~ms}$ and with channel error probability to understand the performance of Abis interface. Figure 5 shows the simulation trace file generated over the NS2 for T200 $=900 \mathrm{~ms}$.

Following peace of trace file shows the performance of Abis over Satellite with T200 = $900 \mathrm{~ms}$ : + enqueue, dequeue, $r$ - received, 1- BTS, 0- Satellite, 2- BSC. Trace file is depicted in the following sequence.

Frame state - time - from - to - LAPD - byte - via source - destination - N(S) - N(R) - longitude - latitude frame

The call set-up time is given by

$$
t=N * t^{\prime}=30 * 0.334=10.02 \text {. }
$$

$N$ : number of command and response I frames, and $t^{\prime}=$ Satellite delay + processing delay. Typical values obtained from the simulation are $N=30, t^{\prime}=33 \mathrm{msec}$, which amounts to $t=10.02 \mathrm{sec}$.

Figure 6 shows that for lower timer values, the continuous retransmission may cause link congestion and will delay call set-up time $t=10.03$ for $T 200=900 \mathrm{~ms}$ and $t=10.42$ for $\mathrm{T} 200=200 \mathrm{~ms}$.

Hence the optimum value of the T200 retransmission timer has to be setup based on the actual network (satellite + processing) delay experienced.

The call set-up time delay due to lost I frame is given by

$$
t=N l * T 200=1 * 900 \mathrm{~ms} .
$$

$N l$ : Number of lost I-frame.

The General equation for call set-up time can be written as

$$
t=N * t^{\prime}+N l * T 200
$$

Figure 7 shows the trace generated in NS2 under channel error probability of $7 \%$. From the figure, it can be concluded that continuous retransmission will cause high link congestion, and service availability will be reduced as the TCH call set-up time will not increase dramatically. This

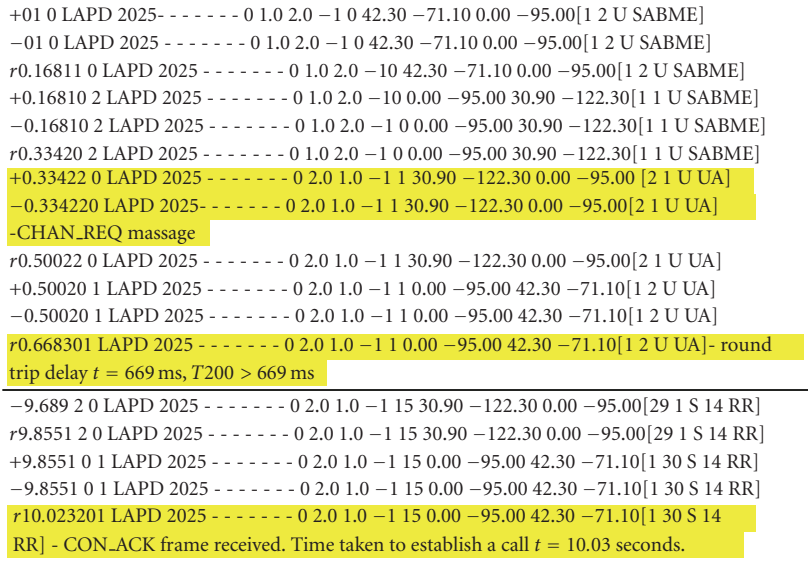

FIgURE 5: Shows the TCH call set-up time for T200 $=900 \mathrm{~ms}$.

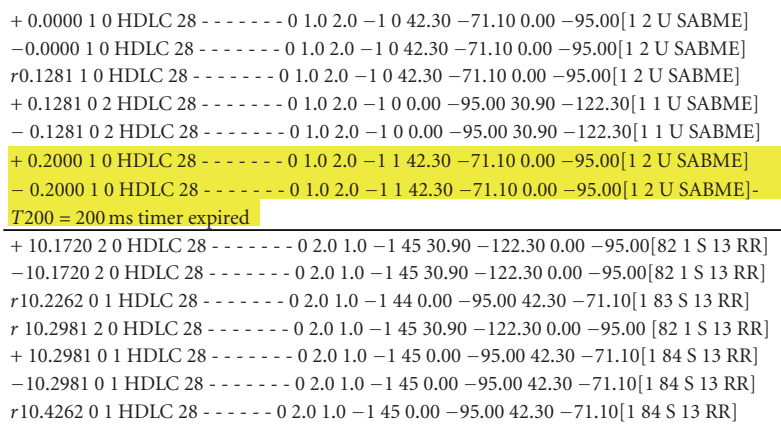

Figure 6: TCH call set up time T200 = $200 \mathrm{~ms}$.

is because retransmitted I frame will receive response of the first sent I frame. However, under the channel errors, the call set-up time increases due to the actual loss of I frames. Table 2 below gives call set-up time obtained from the simulation under various scenarios.

4.2.1. Comparison of Various Network Scenarios and Network Optimizations. Different scenarios are considered to find optimal network configuration for Abis over DVB-S2 platform Table 3. The number of TRX is varied from 1 to 21. Subsequently, the number of users and data rates on Abis interface and Satellite channel is changed. During simulation FEC 3/4 is considered.

Data rate evaluation is done under different data rate definitions.

(1) Abis data rate. Abis data rate corresponds to the data rate after idle time slot elimination. It depends on TRX quantity.

(2) Useful data rate. This is the occupied TS of Abis data by one user. This corresponds to one occupied voice channel by each subscriber.

(3) Satellite data rate. This is the time interval between consecutive sent DVB-S2 frames. DVB-S2 frame is 16200 bit. For, for example, sending DVB-S2 frame, each $40 \mathrm{~ms}$ will give $405 \mathrm{Kbps}$ satellite data rate. 
TABLE 2: Call set-up time for different scenarios.

\begin{tabular}{lc}
\hline Scenario & Value (seconds) \\
\hline$T=900 \mathrm{~ms}$ & 10.02 \\
$T=200 \mathrm{~ms}$ & 10.42 \\
$T=900 \mathrm{~ms}+7 \%$ loss & 10.92 \\
\hline
\end{tabular}

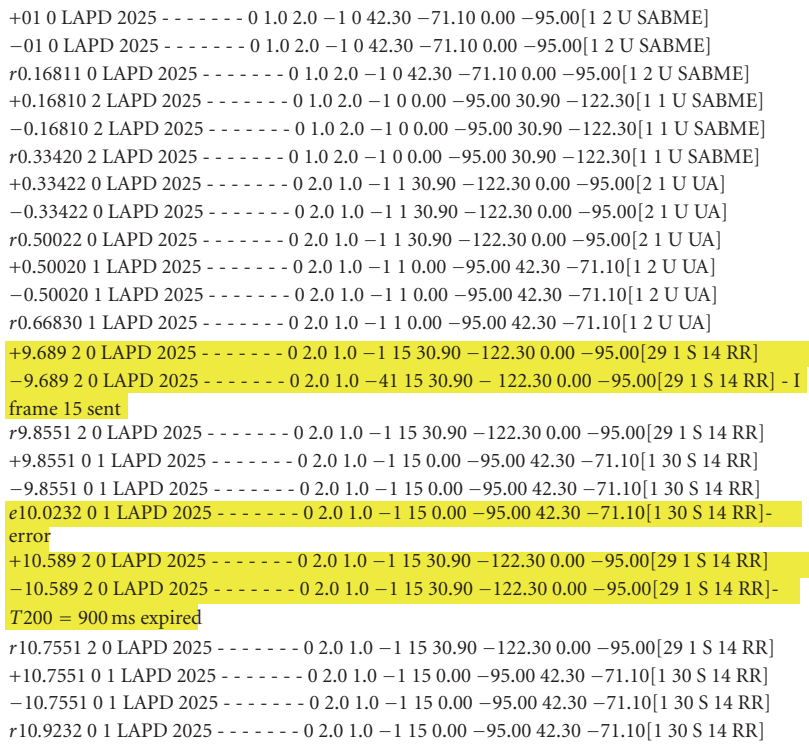

Figure 7: TCH call set-up time with channel error probability 7\%.

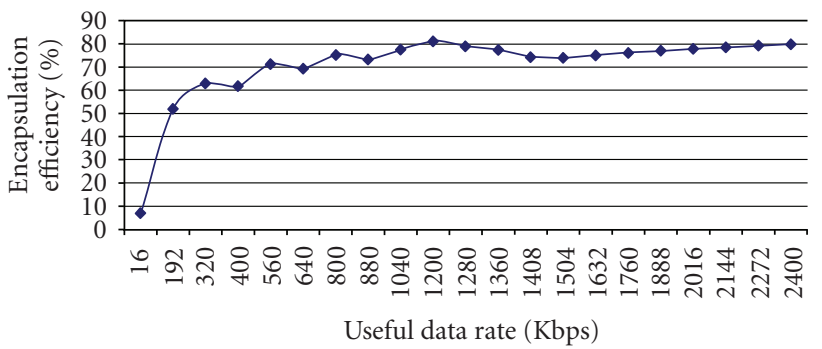

FIGURE 8: Encapsulation efficiency versus useful data rate for DVBS2 short frames.

From Figure 8, we can observe that configuration of one TRX will have poor encapsulation efficiency. With increasing of Abis data rate encapsulation efficiency improves and varies for different data rates. Figure 9 shows Encapsulation efficiency versus useful data rate for DVB-S2 long frames.

In comparison to DVB-S2 short frames, long frames have almost the same encapsulation efficiency. One disadvantage of DVB-S2 long frames is higher delay.

Figure 10 shows delay versus useful data rate for DVB-S2 short and long frames.

From Figure 10, we can see that in case of one TRX and one occupied TCH delay between transferring DVB-S2 frame will reach $200 \mathrm{~ms}$ for long and $50 \mathrm{~ms}$ for short frames. Delay between transmissions of DVB-S2 frames will reduce with increase of data rate on Satellite channel and on Abis interface.

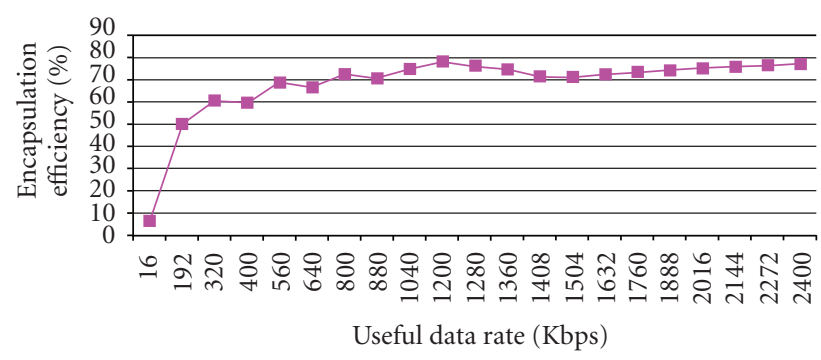

Figure 9: Encapsulation efficiency versus useful data rate for DVBS2 long frames.

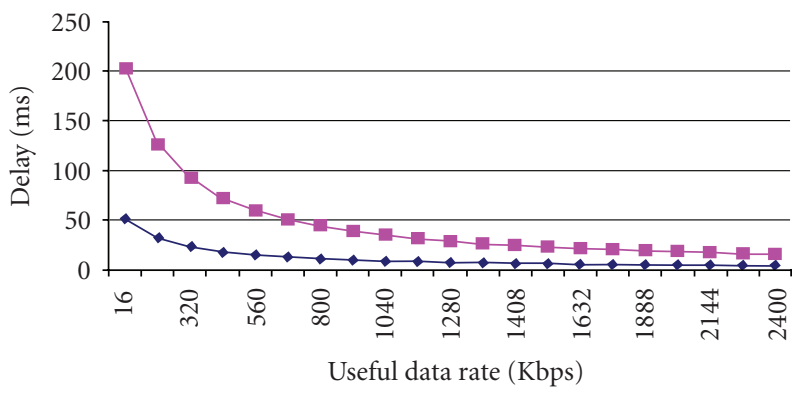

Figure 10: Delay versus useful data rate for DVB-S2 short and long frames.

\section{Link Budget Estimations for DVB-S2 Platform}

Link budget estimations are done considering GSAT-3 (http://www.isro.org/) satellite parameters. DVB-S2 platform has 28 modulation coding (ModCode) modes. Table 4 shows required energy per transmitted symbol $E_{s} / N_{0}(\mathrm{~dB})$ is the figure obtained from computer simulations.

From (9), the required $E_{b} / N_{o}$ for each ModCode can be calculated

$$
\frac{E_{b}}{N_{0}}=\frac{E_{s}}{N_{0}}-10 \log \left(n_{\text {tot }}\right)
$$

where $\eta_{\text {tot }}$ is spectral efficiency.

$\mathrm{C} / \mathrm{N}_{0}$ total can be found from (10).

$$
\left(\frac{C}{N_{0}}\right)=\frac{E_{b}}{N_{0}}+10 \log \left(R_{b}\right)(\mathrm{dBHz}) .
$$

$R_{b}$ is Information rate (Bits/s).

DVB-S2 platform can support three Roll Off factor modes 0.35, 0.25, and 0.20. During Link Budget estimation, value of 0.25 is considered.

Different scenarios are evaluated with different numbers of BTS and TRX's within BTS. Table 5 shows all three scenarios.

Link Budget Estimations are done considering idle time slot elimination technique.

5.1. Results and Analysis. Table 6 shows obtained results for all DVB-S2 modulation modes with FEC 3/4, for a single BTS connection point-to-point SCPC link. 
TABLE 3: Different network parameters during simulation.

\begin{tabular}{|c|c|c|c|c|c|c|}
\hline Number of TRX & DFL short frame bit & DFL long frame bit & Abis DR Kbps & MS & Useful data rate Kbps & Satellite data rate Kbps \\
\hline 1 & 11712 & 48408 & 320 & 1 & 16 & 400 \\
\hline 2 & 11712 & 48408 & 512 & 12 & 192 & 592 \\
\hline 3 & 11712 & 48408 & 704 & 20 & 320 & 784 \\
\hline 4 & 11712 & 48408 & 896 & 25 & 400 & 976 \\
\hline 5 & 11712 & 48408 & 1088 & 35 & 560 & 1168 \\
\hline 6 & 11712 & 48408 & 1280 & 40 & 640 & 1360 \\
\hline 7 & 11712 & 48408 & 1472 & 50 & 800 & 1552 \\
\hline 8 & 11712 & 48408 & 1664 & 55 & 880 & 1744 \\
\hline 9 & 11712 & 48408 & 1856 & 65 & 1040 & 1936 \\
\hline 10 & 11712 & 48408 & 2048 & 75 & 1200 & 2128 \\
\hline 11 & 11712 & 48408 & 2240 & 80 & 1280 & 2320 \\
\hline 12 & 11712 & 48408 & 2432 & 85 & 1360 & 2512 \\
\hline 13 & 11712 & 48408 & 2624 & 88 & 1408 & 2704 \\
\hline 14 & 11712 & 48408 & 2816 & 94 & 1504 & 2896 \\
\hline 15 & 11712 & 48408 & 3008 & 102 & 1632 & 3088 \\
\hline 16 & 11712 & 48408 & 3200 & 110 & 1760 & 3280 \\
\hline 17 & 11712 & 48408 & 3392 & 118 & 1888 & 3472 \\
\hline 18 & 11712 & 48408 & 3584 & 126 & 2016 & 3664 \\
\hline 19 & 11712 & 48408 & 3776 & 134 & 2144 & 3856 \\
\hline 20 & 11712 & 48408 & 3968 & 142 & 2272 & 4048 \\
\hline 21 & 11712 & 48408 & 4160 & 150 & 2400 & 4240 \\
\hline
\end{tabular}

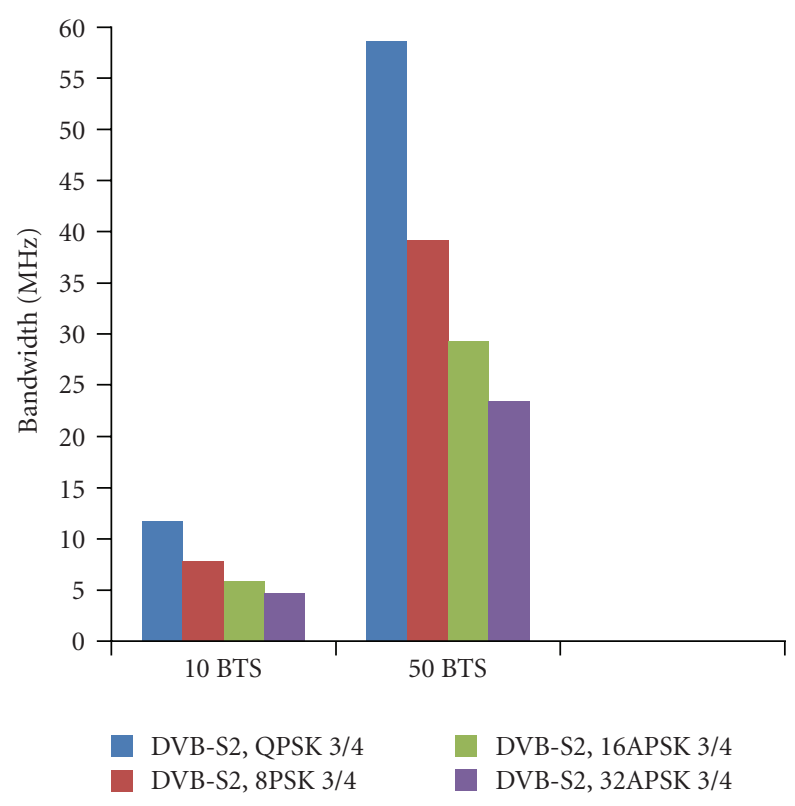

FIgURE 11: Required bandwidth for 10 and 50 BTS.

Figure 11 gives a comparison between two scenarios considering 10 and 50 BTS for DVB-S2 platform.

The cost of bandwidth on Satellite is taken as 4000 US $\$$ per $1 \mathrm{MHz}$ per month [12]. Since Satellite is distance insensitive, this cost will be constant, in comparison to terrestrial scenario where cost of the $2.048 \mathrm{Mbps}$ E1 channel is distance-dependent.
TABle 4: $E_{S} / N_{0}$ performance at Quasi-Error-Free PER $=10^{-7}$ (AWGN channel).

\begin{tabular}{lcc}
\hline Mode & Spectral efficiency $\left(\eta_{\text {tot }}\right)$ & $\begin{array}{c}\text { Ideal } E_{s} / N_{0}(\mathrm{~dB}) \text { for } \\
\text { FECFRAME length }=64 \\
800 \text { bit }\end{array}$ \\
\hline QPSK 3/4 & 1,487473 & 4,03 \\
8PSK 3/4 & 2,228124 & 7,91 \\
16APSK 3/4 & 2,966728 & 10,21 \\
32APSK 3/4 & 3,703295 & 12,73 \\
\hline
\end{tabular}

TABLE 5: Number of TRX for each case.

\begin{tabular}{lccc}
\hline & Single BTS & 10 BTS & 50 BTS \\
\hline No of TRX & $8,4,1$ & 3 & 3 \\
\hline
\end{tabular}

Figure 12 illustrates cost of bandwidth per month for single BTS configuration with different number of TRX.

Figure 13 shows a comparison of different scenarios of 10 and 50 BTS configuration with 3 TRX in each BTS.

\section{Conclusions}

During studies of Abis interface, it was found that one of the weaknesses of this interface is that there is no network layer to serve number of BTSs which are located in different areas within the network and connected via Geostationary Satellite. 
TABLE 6: Required bandwidth for all modulation modes of DVB-S2 for single BTS configuration.

\begin{tabular}{lcccc}
\hline & & SCPC, 8TRX & SCPC, 4TRX & SCPC, TRX \\
\hline Information Rate & $\mathrm{kbps}$ & 1664 & 896 & 746,6 \\
Occupied RF bandwidth (QPSK) & $\mathrm{KHz}$ & 1386,6 & 497,77 & 266,6 \\
Occupied RF bandwidth (8PSK) & $\mathrm{KHz}$ & 924,44 & 373,33 & 177,77 \\
Occupied RF bandwidth (16APSK) & $\mathrm{KHz}$ & 693,33 & 298,66 & 133,33 \\
Occupied RF bandwidth (32APSK) & $\mathrm{KHz}$ & 554,66 & 106,66 \\
\hline
\end{tabular}

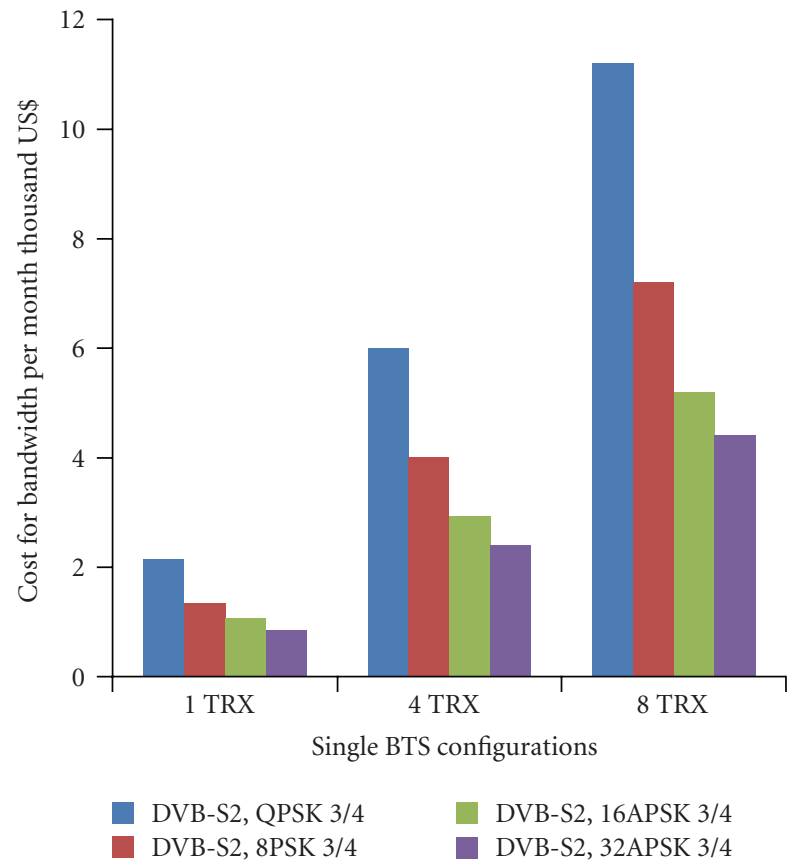

FIGURE 12: Required cost for bandwidth for single BTS configuration.

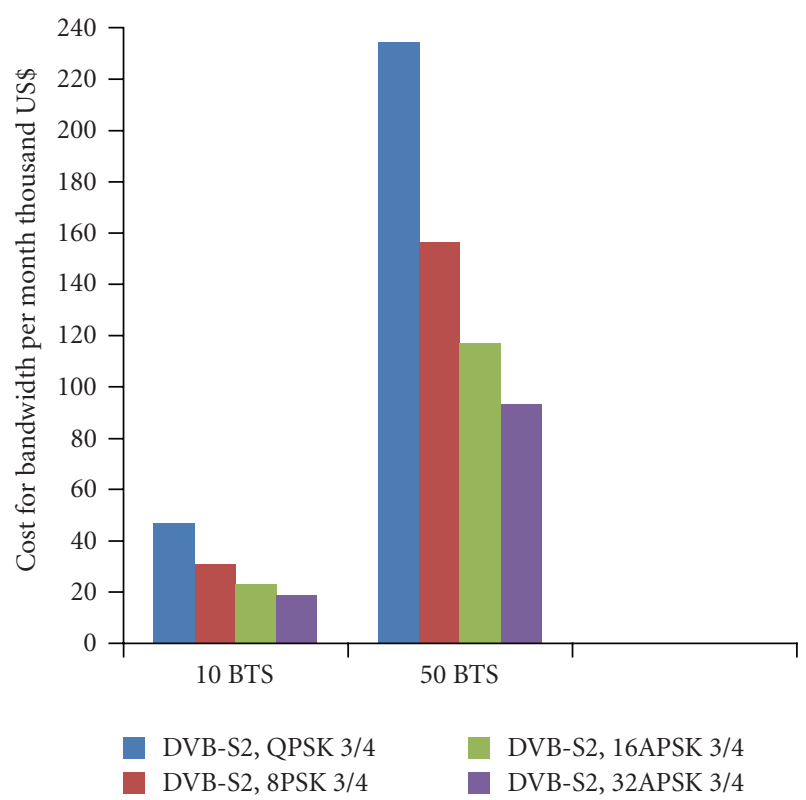

FIGURE 13: Required cost for bandwidth with more number of BTS.
Traffic and subscriber density calculations show that more numbers of SDCCH and TCH channels should be configured to serve the same amount of MS's as in terrestrial scenario.

From the above results, it can be concluded that continuous retransmission of LAPD frames will cause high link congestion which is due to small value of T200. Frame loss will delay call set-up time. From simulation, it is observed that round trip delay for DVB-S2 short frames will reach $334 \mathrm{~ms}$. It is suggested to set the number of retransmissions counter N200 to the maximum value in Abis over Satellite to overcome losses on Satellite channel. It is observed that increase of number of TRX and useful data rate will improve encapsulation efficiency, and Satellite resources will be utilized more efficiently while using DVBS2 platform. It is also observed that DVB-S2 long frames are not suitable because of higher delay between retransmissions. When the number of TRX reaches 10 and the number of MS 75 encapsulation efficiency reaches to $80 \%$, delay between transmitting DVB-S2 short frames will reach $8 \mathrm{~ms}$ which is acceptable for Satellite environment.

Simulation results on DVB-S2 encapsulation show that different ModCode modes will give different encapsulation efficiencies. For efficient encapsulation into DVB-S2 frame, appropriate ModCode mode should be chosen; however, the selection of the ModCode is dependent on the channel fades encountered. It is concluded that DVB-S2 will provide several advantages like improved Roll-Off factor, different power and bandwidth efficient modulation modes. For efficient utilization of frequency spectrum parameters such as padding efficiency and overall encapsulation efficiencies should be evaluated.

Present development of $3 \mathrm{G}$ and $4 \mathrm{G}$ systems in the world market shows that requirement for new services and higher data rates will grow. 3G (WCDMA/UMTS) which offers $2 \mathrm{M}$ of data rate per subscriber is a good gateway in the world of Data and Internet. Hence it is important to know how these technologies may be provided in the remote areas efficiently for 24 hours in a day.

Paper addressed only GSM technology. Future work for 3G, 4G technologies should be done.

\section{References}

[1] ETSI EN 302307 version1.1.1, “Digital Video Broadcasting (DVB); Second generation framing structure, channel coding and modulation systems for Broadcasting, Interactive Services, News Gathering and other broadband satellite applications," 2004. 
[2] S. B. Musabekov, P. K. Srinivasan, A. S. Durai, and R. R. Ibroimov, "Simulation analysis of abis interface over IP over DVB-S2-RCS in a GSM over satellite network," in Proceedings of the 4th IEEE/IFIP International Conference in Central Asia on Internet (ICI '08), September 2008.

[3] The VINT Project, "The ns Manual (formerly ns Notes and Documentation)," July 2008.

[4] DVB Document A134, "Generic Stream Encapsulation (GSE) Implementation Guidelines," February 2009.

[5] Ses New Skies B.V., "GSM Over Satellite," 2006.

[6] 3GPP TS 08.58 version 8.6.0, "Base Station Controller-Base Transceiver Station (BCS-BTS) Interface Layer 3 Specification," http://www.3gpp.org/.

[7] 3GPP TS 08.56 version 8.0.1, "BSC-BTS Layer 2; Specification," http://www.3gpp.org/.

[8] 3GPP TS 08.54 version 8.0.1, "BSC-BTS Layer 1; Structure of Physical Circuits," http://www.3gpp.org/.

[9] ETSI EN 300 737, "Digital cellular telecommunication systems (Phase2+) In-band control of remote transcoders and rate adaptors for full rate traffic channels," V7.2.1, September 2000.

[10] S. B. Musabekov and R. R. Ibraimov, "NS-2 network performance evaluation of abis interface over DVB-S2 in the GSM over satellite network," in Proceedings of the 1st Asian Himalayas International Conference on Internet (AH-ICI '09), pp. 1-5, 2009.

[11] ETS 300 125, “Integrated Services Digital Network (ISDN);User-network interface data link layer specification; Application of CCITT Recommendations Q.920/I.440 and Q.921/I.441,” part-2, subclause 5.9.1, September 1991.

[12] Globecomm, "Optimizing GSM Abis ExtensionsVia Satellite," 2006. 

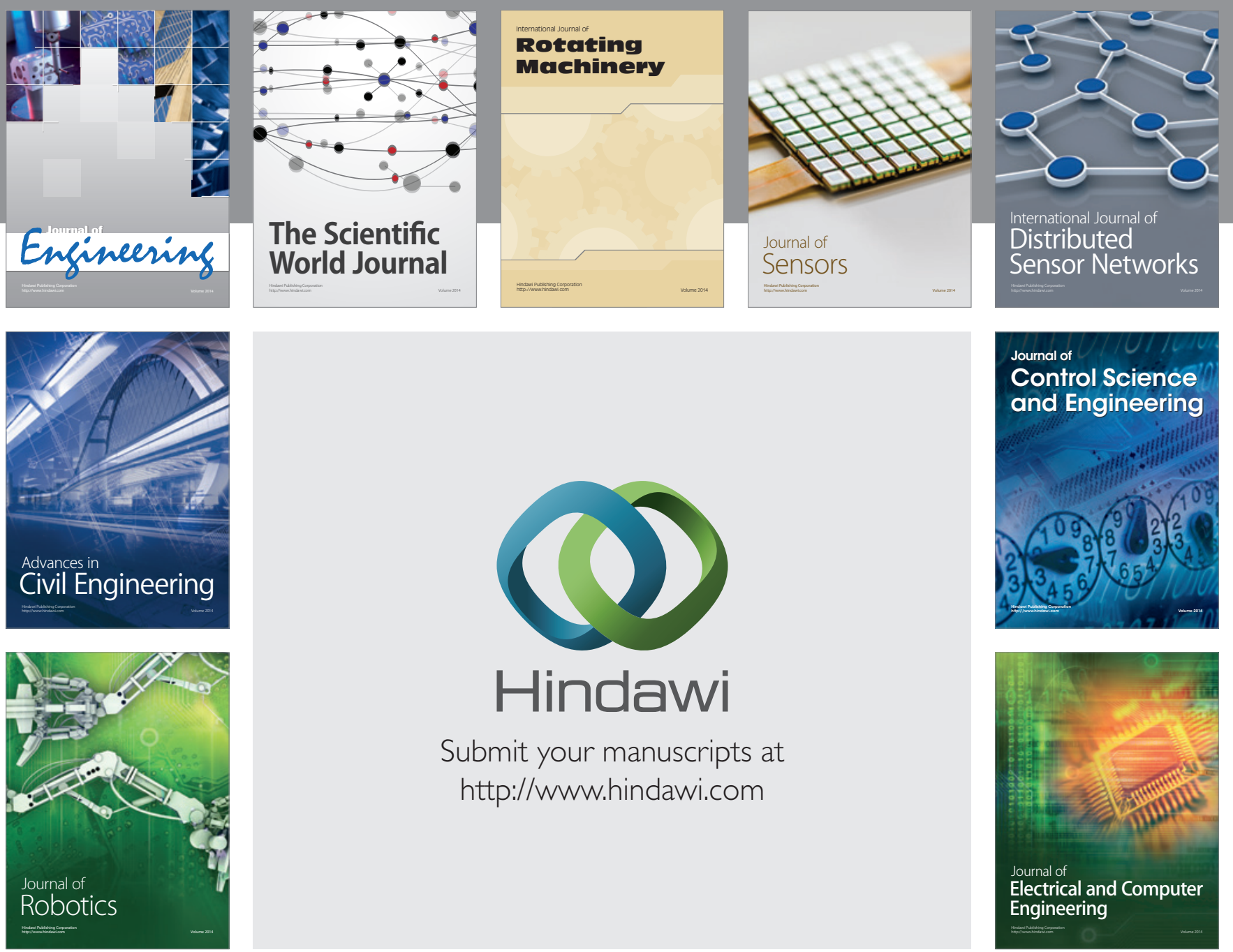

Submit your manuscripts at

http://www.hindawi.com
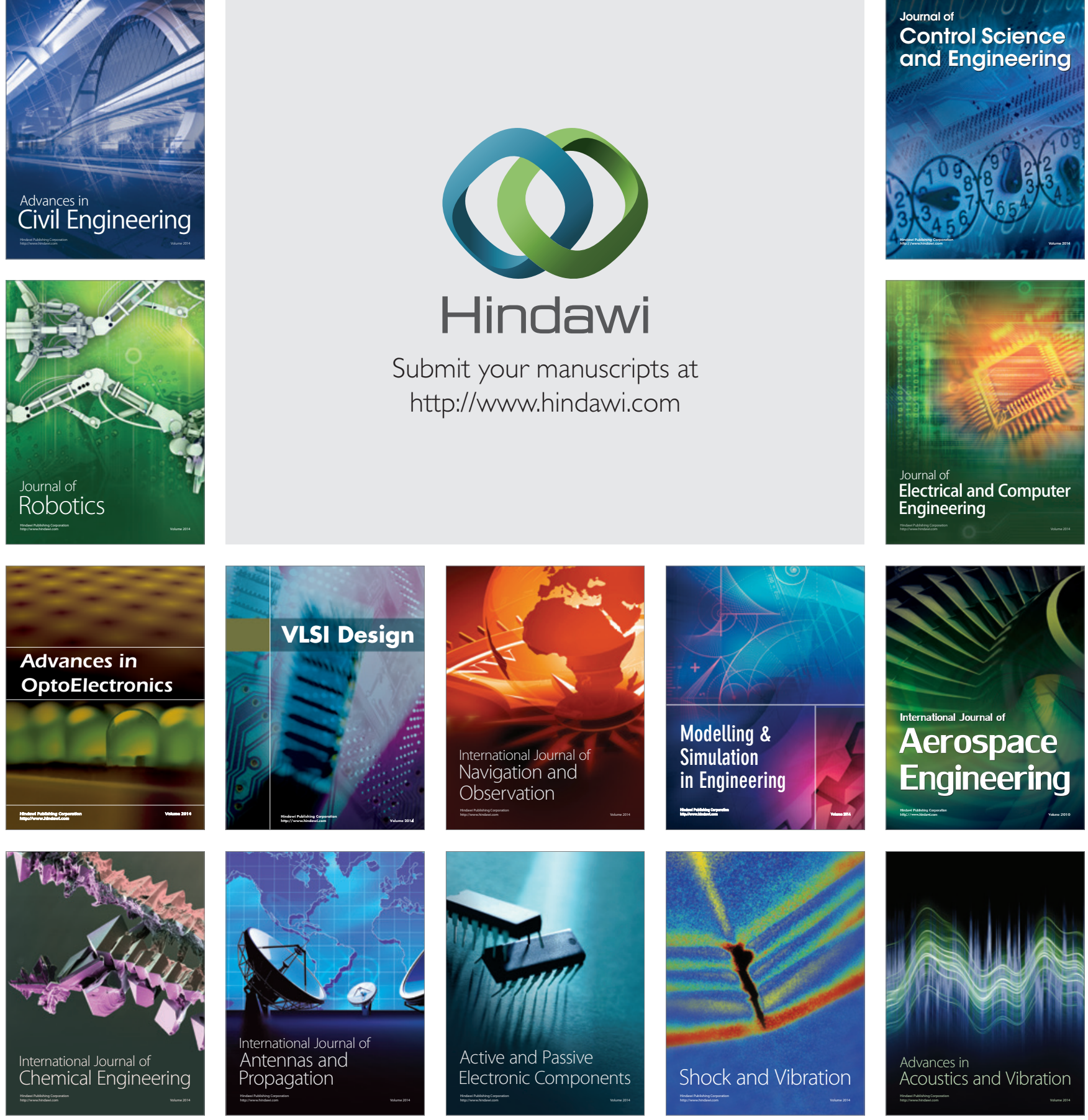